\title{
On the Difference of Coefficients of Bazilevič Functions
}

\author{
Nak Eun Cho ${ }^{1}$ Y Young Jae $\mathrm{Sim}^{2} \cdot$ Derek K. Thomas $^{3}$
}

Received: 1 February 2019 / Revised: 26 June 2019 / Accepted: 16 July 2019 /

Published online: 28 September 2019

(c) The Author(s) 2019

\section{Abstract}

Let $f$ be analytic in the unit disk $\mathbb{D}=\{z \in \mathbb{C}:|z|<1\}$, and $\mathcal{S}$ be the subclass of normalized univalent functions given by $f(z)=z+\sum_{n=2}^{\infty} a_{n} z^{n}$ for $z \in \mathbb{D}$. We give bounds for ||$a_{3}|-| a_{2}||$ for the subclass $\mathcal{B}(\alpha, i \beta)$ of generalized Bazilevič functions when $\alpha \geq 0$, and $\beta$ is real.

Keywords Univalent function - Close-to-convex function · Bazilevič function · Difference of coefficients

Mathematics Subject Classification 30C45 $\cdot 30 \mathrm{C} 50 \cdot 30 \mathrm{C} 55$

\section{Introduction}

Let $\mathcal{A}$ denote the class of analytic functions $f$ in the unit disk $\mathbb{D}=\{z \in \mathbb{C}:|z|<1\}$ normalized by $f(0)=0=f^{\prime}(0)-1$. Then for $z \in \mathbb{D}, f \in \mathcal{A}$ has the following representation

$$
f(z)=z+\sum_{n=2}^{\infty} a_{n} z^{n} .
$$

\section{Communicated by Stephan Ruscheweyh.}

$凶 \quad$ Derek K. Thomas

d.k.thomas@swansea.ac.uk

Nak Eun Cho

necho@pknu.ac.kr

Young Jae Sim

yjsim@ks.ac.kr

1 Department of Applied Mathematics, Pukyong National University, Busan 48513, Korea

2 Department of Mathematics, Kyungsung University, Busan 48434, Korea

3 Department of Mathematics, Swansea University, Bay Campus, Swansea SA1 8EN, UK 
Let $\mathcal{S}$ denote the subclass of all univalent (i.e., one-to-one) functions in $\mathcal{A}$.

In 1985, de Branges [2] solved the famous Bieberbach conjecture by showing that if $f \in \mathcal{S}$, then $\left|a_{n}\right| \leq n$ for $n \geq 2$, with equality when $f(z)=k(z):=z /(1-z)^{2}$, or a rotation. It was therefore natural to ask if for $f \in \mathcal{S}$, the inequality ||$a_{n+1}|-| a_{n}|| \leq 1$ is true when $n \geq 2$. This was shown not to be the case even when $n=2$ [4], and that the following sharp bounds hold.

$$
-1 \leq\left|a_{3}\right|-\left|a_{2}\right| \leq \frac{3}{4}+e^{-\lambda_{0}}\left(2 e^{-\lambda_{0}}-1\right)=1.029 \ldots,
$$

where $\lambda_{0}$ is the unique value of $\lambda$ in $0<\lambda<1$, satisfying the equation $4 \lambda=e^{\lambda}$.

Hayman [6] showed that if $f \in \mathcal{S}$, then ||$a_{n+1}|-| a_{n}|| \leq C$, where $C$ is an absolute constant. The exact value of $C$ is unknown, best estimate to date being $C=3.61 \ldots$ [5], which because of the sharp estimate above when $n=2$, cannot be reduced to 1 .

Denote by $\mathcal{S}^{*}$ the subclass of $\mathcal{S}$ consisting of starlike functions, i.e. functions $f$ which map $\mathbb{D}$ onto a set which is star-shaped with respect to the origin. Then it is well-known that a function $f \in \mathcal{S}^{*}$ if, and only if, for $z \in \mathbb{D}$

$$
\operatorname{Re}\left\{\frac{z f^{\prime}(z)}{f(z)}\right\}>0 \text {. }
$$

It was shown in [8], that when $f \in \mathcal{S}^{*}$, then ||$a_{n+1}|-| a_{n}|| \leq 1$ is true when $n \geq 2$.

Next denote by $\mathcal{K}$ the subclass of $\mathcal{S}$ consisting of functions which are close-toconvex, i.e. functions $f$ which map $\mathbb{D}$ onto a close-to-convex domain. Then again it is well-known that a function $f \in \mathcal{K}$ if, and only if, there exists $g \in \mathcal{S}^{*}$ such that for $z \in \mathbb{D}$

$$
\operatorname{Re}\left\{\frac{z f^{\prime}(z)}{g(z)}\right\}>0
$$

Koepf [7] showed that if $f \in \mathcal{K}$, then ||$a_{n+1}|-| a_{n}|| \leq 1$, when $n=2$, but establishing this inequality when $n \geq 3$ remains an open problem.

In 1955, Bazilevič [1] extended the notion of starlike and close-to-convex functions by showing that if $f \in \mathcal{A}$, and is given by (1.1), then if $\alpha>0$ and $\beta \in \mathbb{R}, f$ given by

$$
f(z)=\left((\alpha+i \beta) \int_{0}^{z} g^{\alpha}(t) p(t) t^{i \beta-1} d t\right)^{1 /(\alpha+i \beta)},
$$

where $g \in \mathcal{S}^{*}$, and $p \in \mathcal{P}$, the class of functions with positive real part in $\mathbb{D}$, then functions defined by (1.3) form a subset of $\mathcal{S}$. Such functions are known as Bazilevič functions.

We note that in the original definition of Bazilevič functions [1], Bazilevič assumed that $\alpha>0$, however Sheil-Small [10], subsequently showed that when $\alpha=0$, such functions also belong to $\mathcal{S}$, and satisfy

$$
\frac{z f^{\prime}(z)}{f(z)}\left(\frac{f(z)}{z}\right)^{i \beta}=p(z)
$$

where $p \in \mathcal{P}$. 
For $\alpha \geq 0$ and $\beta \in \mathbb{R}$, we denote functions defined as in (1.3) and (1.4) by $\mathcal{B}(\alpha, i \beta)$, and note that the class $\mathcal{B}(\alpha, 0) \equiv \mathcal{B}(\alpha)$ has been extensively studied, and that $\mathcal{B}(0,0) \equiv \mathcal{S}^{*}$ and $\mathcal{B}(1,0) \equiv \mathcal{K}$.

Another well studied subclass of $\mathcal{B}(\alpha, i \beta)$ is the class $\mathcal{B}_{1}(\alpha, i \beta)$, where $\beta=0$ and the starlike function $g(z) \equiv z$, (see e.g. [11]). This class is usually denoted by $\mathcal{B}_{1}(\alpha)$. Although much is known about the initial coefficients of functions in $\mathcal{B}_{1}(\alpha)$, there appears to be no published information concerning the difference of coefficients. We also note that $\mathcal{B}_{1}(1,0)$ reduces to the class of functions in $\mathcal{R}$ such that their derivatives have positive real part for $z \in \mathbb{D}$, and that the class $\mathcal{B}_{1}(1, i \beta)$ has been little studied.

In this paper we present some inequalities for ||$a_{3}|-| a_{2}||$ when $f \in \mathcal{B}(\alpha, i \beta)$, obtaining sharp bounds when $f \in \mathcal{B}(\alpha)$, and $f \in \mathcal{B}_{1}(\alpha, i \beta)$ when $\alpha \geq 0$ and $\beta \in \mathbb{R}$. We also give the sharp bounds for ||$a_{3}|-| a_{2}||$, when $f \in \mathcal{B}(0, i \beta)$.

\section{Preliminary Lemmas}

Denote by $\mathcal{P}$, the class of analytic functions $p$ with positive real part on $\mathbb{D}$ given by

$$
p(z)=1+\sum_{n=1}^{\infty} p_{n} z^{n} .
$$

We will use the following properties for the coefficients of functions $\mathcal{P}$, given by (2.1).

Lemma 2.1 [9] For $p \in \mathcal{P}$ and $v \in \mathbb{C}$,

$$
\left|p_{2}-\frac{v}{2} p_{1}^{2}\right| \leq 2 \max \{|v-1| ; 1\},
$$

and

$$
\left|p_{2}-\frac{1}{2} p_{1}^{2}\right| \leq 2-\frac{1}{2}\left|p_{1}\right|^{2} .
$$

Both inequalities are sharp.

Lemma 2.2 [3] If $p \in \mathcal{P}$, then

$$
p_{1}=2 \zeta_{1}
$$

and

$$
p_{2}=2 \zeta_{1}^{2}+2\left(1-\left|\zeta_{1}\right|^{2}\right) \zeta_{2}
$$

for some $\zeta_{i} \in \overline{\mathbb{D}}, i \in\{1,2\}$. For $\zeta_{1} \in \mathbb{T}$, the boundary of $\mathbb{D}$, there is a unique function $p \in \mathcal{P}$ with $p_{1}$ as in (2.2), namely, 


$$
p(z)=\frac{1+\zeta_{1} z}{1-\zeta_{1} z} \quad(z \in \mathbb{D})
$$

For $\zeta_{1} \in \mathbb{D}$ and $\zeta_{2} \in \mathbb{T}$, there is a unique function $p \in \mathcal{P}$ with $p_{1}$ and $p_{2}$ as in (2.2) and (2.3), namely,

$$
p(z)=\frac{1+\left(\bar{\zeta}_{1} \zeta_{2}+\zeta_{1}\right) z+\zeta_{2} z^{2}}{1+\left(\bar{\zeta}_{1} \zeta_{2}-\zeta_{1}\right) z-\zeta_{2} z^{2}} \quad(z \in \mathbb{D})
$$

We will also need the following well-known result.

Lemma 2.3 [7, Lem. 3] Let $g \in \mathcal{S}^{*}$ and be given by $g(z)=z+\sum_{n=2}^{\infty} b_{n} z^{n}$. Then for any $\lambda \in \mathbb{C}$,

$$
\left|b_{3}-\lambda b_{2}^{2}\right| \leq \max \{1 ;|3-4 \lambda|\} \text {. }
$$

The inequality is sharp when $g(z)=k(z)$ if $|3-4 \lambda| \geq 1$, and when $g(z)=\left(k\left(z^{2}\right)\right)^{1 / 2}$ if $|3-4 \lambda|<1$.

\section{The class $\mathcal{B}(\alpha, i \beta)$}

We begin by proving the following inequalities for $f \in \mathcal{B}(\alpha, i \beta)$.

Theorem 3.1 Let $f \in \mathcal{B}(\alpha, i \beta)$ and be given by (1.1). If $0 \leq \alpha \leq(\sqrt{17}-1) / 2$ and $\beta \in \mathbb{R}$, then

$$
-1 \leq\left|a_{3}\right|-\left|a_{2}\right| \leq \frac{2+\alpha}{|2+\alpha+i \beta|}
$$

Proof Recall that $\left|a_{2}\right|-\left|a_{3}\right| \leq 1$ for all $f \in \mathcal{S}[4$, Thm. 3.11]. So, since $\mathcal{B}(\alpha, i \beta) \subset \mathcal{S}$ for all $\alpha \geq 0$ and $\beta \in \mathbb{R}$, it is sufficient to prove the upper bound in (3.1).

Let $f \in \mathcal{B}(\alpha, i \beta)$ be of the form (1.1). Then from (1.3) we have

$$
\left(\frac{z f^{\prime}(z)}{f(z)}\right)\left(\frac{f(z)}{g(z)}\right)^{\alpha}\left(\frac{f(z)}{z}\right)^{i \beta}=p(z),
$$

for some $g \in \mathcal{S}^{*}$ and $p \in \mathcal{P}$. Writing

$$
g(z)=z+\sum_{n=2}^{\infty} b_{n} z^{n} \text { and } p(z)=1+\sum_{n=1}^{\infty} p_{n} z^{n}
$$

and equating the coefficients, we obtain

$$
a_{2}=\frac{\alpha b_{2}+p_{1}}{1+\alpha+i \beta}
$$


and

$$
\begin{aligned}
a_{3}= & \frac{p_{2}}{2+\alpha+i \beta}-\frac{(-1+\alpha+i \beta) p_{1}^{2}}{2(1+\alpha+i \beta)^{2}}+\frac{\alpha(3+\alpha+i \beta) b_{2} p_{1}}{(1+\alpha+i \beta)^{2}(2+\alpha+i \beta)} \\
& +\frac{\alpha b_{3}}{2+\alpha+i \beta}+\frac{\alpha\left(-1+\alpha-2 i \beta-i \alpha \beta+\beta^{2}\right) b_{2}^{2}}{2(2+\alpha+i \beta)(1+\alpha+i \beta)^{2}}
\end{aligned}
$$

Let $\mu_{1}=(3+\alpha+i \beta) /(2(2+\alpha+i \beta))$, and suppose that $\left|a_{2}\right| \leq 1 /\left|\mu_{1}\right|$. Then by Lemmas 2.1 and 2.3 we have

$$
\begin{aligned}
\left|a_{3}-\mu_{1} a_{2}^{2}\right| & =\left|\frac{1}{2+\alpha+i \beta}\left(p_{2}-\frac{1}{2} p_{1}^{2}+\alpha\left(b_{3}-\frac{1}{2} b_{2}^{2}\right)\right)\right| \\
& \leq \frac{2+\alpha}{|2+\alpha+i \beta|} .
\end{aligned}
$$

Thus from (3.4) we obtain

$$
\left|a_{3}\right|-\left|a_{2}\right| \leq\left|a_{3}\right|-\left|\mu_{1}\right|\left|a_{2}\right|^{2} \leq\left|a_{3}-\mu_{1} a_{2}^{2}\right| \leq \frac{2+\alpha}{|2+\alpha+i \beta|}
$$

Now assume that $1 /\left|\mu_{1}\right| \leq\left|a_{2}\right| \leq 2$, and let $\mu_{2}=1 /(2+\alpha+i \beta)$. Then

$$
a_{3}-\mu_{2} a_{2}^{2}=\Psi_{1}+\frac{1}{2+\alpha+i \beta} \Psi_{2}
$$

where

$$
\Psi_{1}=\frac{\alpha b_{3}}{2+\alpha+i \beta}-\frac{\alpha(1+i \beta) b_{2}^{2}}{2(1+\alpha+i \beta)(2+\alpha+i \beta)},
$$

and

$$
\Psi_{2}=\frac{\alpha b_{2} p_{1}}{(1+\alpha+i \beta)}-\frac{(\alpha+i \beta) p_{1}^{2}}{2(1+\alpha+i \beta)}+p_{2}
$$

Put $\mu=(1+i \beta) /(2(1+\alpha+i \beta))$. Then it is easily seen that $|3-4 \mu|=\mid 1+3 \alpha$ $+i \beta|/| 1+\alpha+i \beta \mid \geq 1$. Thus Lemma 2.3 gives

$$
\left|\Psi_{1}\right| \leq \frac{\alpha}{|2+\alpha+i \beta|}|3-4 \mu|=\frac{\alpha|1+3 \alpha+i \beta|}{|2+\alpha+i \beta||1+\alpha+i \beta|} .
$$

Next use (2.2) and (2.3) in Lemma 2.2 to obtain

$$
\Psi_{2}=\frac{2 \alpha b_{2} \zeta_{1}}{1+\alpha+i \beta}+\frac{2 \zeta_{1}^{2}}{1+\alpha+i \beta}+2\left(1-\left|\zeta_{1}\right|^{2}\right) \zeta_{2},
$$


where $\zeta_{i} \in \overline{\mathbb{D}}(i=1,2)$. The triangle inequality and $\left|b_{2}\right| \leq 2$ then gives

$$
\left|\Psi_{2}\right| \leq \psi\left(\left|\zeta_{1}\right|\right)
$$

where

$$
\psi(x)=2+\frac{4 \alpha}{|1+\alpha+i \beta|} x+2\left(\frac{1-|1+\alpha+i \beta|}{|1+\alpha+i \beta|}\right) x^{2}
$$

with $x \in[0,1]$.

Let $x_{0}=\alpha /(|1+\alpha+i \beta|-1)$, so that $x_{0} \in[0,1]$, and $\psi$ has a unique critical point at $x=x_{0}$. Since $\psi$ has a negative leading coefficient, it follows from (3.7) that for all $x \in[0,1]$,

$$
\left|\Psi_{2}\right| \leq \psi\left(x_{0}\right)=2+\frac{2 \alpha^{2}}{|1+\alpha+i \beta|(|1+\alpha+i \beta|-1)} \quad(x \in[0,1]) .
$$

Therefore from (3.5), (3.6) and (3.10) we obtain

$$
\begin{aligned}
\left|a_{3}-\mu_{2} a_{2}^{2}\right| & \leq \frac{1}{|2+\alpha+i \beta|}\left(2+\frac{\alpha|1+3 \alpha+i \beta|}{|1+\alpha+i \beta|}+\frac{2 \alpha^{2}}{|1+\alpha+i \beta|(|1+\alpha+i \beta|-1)}\right) \\
& =: \Psi(\alpha, \beta) .
\end{aligned}
$$

Next write $y:=\left|a_{2}\right|$, and assume that $y \in\left[1 /\left|\mu_{1}\right|, \tilde{x}\right]$, where

$$
\tilde{x}=\frac{2 \alpha+2}{|1+\alpha+i \beta|}
$$

so that

$$
\left|a_{3}\right|-\left|a_{2}\right| \leq\left|a_{3}-\mu_{2} a_{2}^{2}\right|+\left|\mu_{2}\right|\left|a_{2}\right|^{2}-\left|a_{2}\right| \leq \Psi(\alpha, \beta)+\varphi(y),
$$

where $\varphi$ is defined by

$$
\varphi(y)=\frac{1}{|2+\alpha+i \beta|} y^{2}-y \quad\left(y \in\left[1 /\left|\mu_{1}\right|, \tilde{x}\right]\right) .
$$

Since $\varphi$ is convex on $\left[1 /\left|\mu_{1}\right|, \tilde{x}\right]$,

$$
\varphi(y) \leq \max \left\{\varphi\left(1 /\left|\mu_{1}\right|\right) ; \varphi(\tilde{x})\right\}
$$

for all $y \in\left[1 /\left|\mu_{1}\right|, \tilde{x}\right]$.

Thus in order to establish the upper bound in (3.1), we use (3.10) and (3.11), and need to show that

$$
\Psi(\alpha, \beta)+\varphi\left(\frac{1}{\left|\mu_{1}\right|}\right) \leq \frac{2+\alpha}{|2+\alpha+i \beta|}
$$


and

$$
\Psi(\alpha, \beta)+\varphi(\tilde{x}) \leq \frac{2+\alpha}{|2+\alpha+i \beta|}
$$

We first obtain (3.12).

Since

$$
\frac{4}{|3+\alpha+i \beta|}-2<0 \text { and } \frac{|2+\alpha+i \beta|}{|3+\alpha+i \beta|} \geq \frac{2+\alpha}{3+\alpha}
$$

(3.12) holds provided

$$
\begin{aligned}
A_{1} & :=\frac{\alpha|1+3 \alpha+i \beta|}{|1+\alpha+i \beta|}+\frac{2 \alpha^{2}}{|1+\alpha+i \beta|(|1+\alpha+i \beta|-1)} \\
& +\frac{4(2+\alpha)|2+\alpha+i \beta|}{(3+\alpha)|3+\alpha+i \beta|}-\alpha \\
& \leq \frac{2(2+\alpha)|2+\alpha+i \beta|}{3+\alpha}=: A_{2} .
\end{aligned}
$$

Clearly $A_{1} \leq A_{2}$ is true when $\alpha=0$. For $\alpha>0$, using the inequalities

$$
\frac{|1+3 \alpha+i \beta|}{|1+\alpha+i \beta|} \leq \frac{1+3 \alpha}{1+\alpha}, \quad \frac{1}{|1+\alpha+i \beta|} \leq \frac{1}{1+\alpha}
$$

and

$$
\frac{1}{|1+\alpha+i \beta|-1} \leq \frac{1}{\alpha}
$$

it follows that

$$
\frac{1}{2}\left(A_{1}-A_{2}\right) \leq|2+\alpha+i \beta|\left(\frac{\alpha}{|2+\alpha+i \beta|}+\frac{2(2+\alpha)}{(3+\alpha)|3+\alpha+i \beta|}-\frac{2+\alpha}{3+\alpha}\right) .
$$

We next note that the following is valid provided $\alpha \in[0,(\sqrt{17}-1) / 2]$.

$$
\frac{\alpha}{|2+\alpha+i \beta|}+\frac{2(2+\alpha)}{(3+\alpha)|3+\alpha+i \beta|} \leq \frac{\alpha}{2+\alpha}+\frac{2(2+\alpha)}{(3+\alpha)^{2}} \leq \frac{2+\alpha}{3+\alpha} .
$$

Thus from (3.15) and (3.14), $A_{1} \leq A_{2}$ and (3.12) is established, providing $\alpha \in$ $[0,(\sqrt{17}-1) / 2]$.

Next we prove (3.13), which is satisfied if $B_{1} \leq B_{2}$, where

$$
B_{1}:=\alpha(|1+3 \alpha+i \beta|-|1+\alpha+i \beta|)+\frac{2 \alpha^{2}}{|1+\alpha+i \beta|-1}+\frac{(2 \alpha+2)^{2}}{|1+\alpha+i \beta|}
$$


and

$$
B_{2}:=2(1+\alpha)|2+\alpha+i \beta|
$$

A similar process to the above gives

$$
B_{1} \leq 2 \alpha^{2}+2 \alpha+\frac{(2 \alpha+2)^{2}}{1+\alpha}=2(1+a)(2+a) \leq B_{2}
$$

which proves inequality (3.13), and so the proof of Theorem 3.1 is complete.

When $\beta=0$, we deduce the following, noting that when $\alpha=1$, we obtain the inequality ||$a_{3}|-| a_{2}|| \leq 1$ for $f \in \mathcal{K}$ obtained in [7].

Corollary 3.1 Let $f \in \mathcal{B}(\alpha)$. Then ||$a_{3}|-| a_{2}|| \leq 1$ provided $\left.0 \leq \alpha \leq(\sqrt{17}-1) / 2\right]=$ 1.56 ....

The inequality is sharp when both the functions $f$ and $g$ are the Koebe function.

We end this section by noting from the definition, since $\mathcal{B}_{1}(0, i \beta) \equiv \mathcal{B}(0, i \beta)$, the following is an immediate consequence of Theorem 4.1 below.

Theorem 3.2 Let $f \in \mathcal{B}(0, i \beta)$, and be given by (1.1) with $\beta \in \mathbb{R}$. Then

$$
-\frac{2}{\sqrt{|1+i \beta|^{2}+|3+i \beta|}} \leq\left|a_{3}\right|-\left|a_{2}\right| \leq \frac{2}{|2+i \beta|} .
$$

Both inequalities are sharp.

\section{The class $\mathcal{B}_{1}(\alpha, i \beta)$,}

We next consider the class $\mathcal{B}_{1}(\alpha, i \beta)$, recalling that $f \in \mathcal{B}_{1}(\alpha, i \beta)$ if, and only if, for $\alpha \geq 0$ and $\beta \in \mathbb{R}$,

$$
\operatorname{Re}\left\{\frac{z f^{\prime}(z)}{f(z)}\left(\frac{f(z)}{z}\right)^{\alpha+i \beta}\right\}>0 \quad(z \in \mathbb{D})
$$

We find the sharp upper and lower bounds of $\left|a_{3}\right|-\left|a_{2}\right|$ over the class $\mathcal{B}_{1}(\alpha, i \beta)$.

Theorem 4.1 Let $f \in \mathcal{B}_{1}(\alpha, i \beta)$ for $\alpha \geq 0$ and $\beta \in \mathbb{R}$, and be given by (1.1). Then

$$
-\frac{2}{\sqrt{|1+\alpha+i \beta|^{2}+|3+\alpha+i \beta|}} \leq\left|a_{3}\right|-\left|a_{2}\right| \leq \frac{2}{|2+\alpha+i \beta|} .
$$

Both inequalities are sharp. 
Proof From (3.2), (3.3) (with $b_{2}=b_{3}=0$ ), and Lemma 2.2, we obtain

$$
a_{2}=\frac{2 \zeta_{1}}{1+\alpha+i \beta}
$$

and

$$
a_{3}=\left(\frac{2}{2+\alpha+i \beta}-\frac{2(-1+\alpha+i \beta)}{(1+\alpha+i \beta)^{2}}\right) \zeta_{1}^{2}+\frac{2}{2+\alpha+i \beta}\left(1-\left|\zeta_{1}\right|^{2}\right) \zeta_{2}
$$

for some $\zeta_{i} \in \overline{\mathbb{D}}(i=1,2)$. The triangle inequality gives

$$
\left|a_{3}\right|-\left|a_{2}\right| \leq \psi\left(\left|\zeta_{1}\right|\right)
$$

where

$$
\psi(x)=\kappa_{2} x^{2}+\kappa_{1} x+\kappa_{0} \quad(x \in[0,1])
$$

with

$$
\begin{aligned}
& \kappa_{2}=\left|\frac{2}{2+\alpha+i \beta}-\frac{2(-1+\alpha+i \beta)}{(1+\alpha+i \beta)^{2}}\right|-\frac{2}{|2+\alpha+i \beta|}, \\
& \kappa_{1}=-\frac{2}{|1+\alpha+i \beta|}, \quad \text { and } \quad \kappa_{0}=\frac{2}{|2+\alpha+i \beta|} .
\end{aligned}
$$

We first prove the upper bound in (4.1).

If $\kappa_{2} \leq 0$, then since $\kappa_{1}<0$, we have $\psi^{\prime}(x)=2 \kappa_{2} x+\kappa_{1}<0$ for all $x \in[0,1]$. Thus

$$
\psi(x) \leq \psi(0)=\kappa_{0} \quad(x \in[0,1])
$$

Suppose next that $\kappa_{2}>0$. We now note that $\kappa_{2}+\kappa_{1} \leq 0$, since

$$
\begin{aligned}
\frac{1}{2}\left(\kappa_{2}+\kappa_{1}\right) & \leq \frac{|-1+\alpha+i \beta|}{|1+\alpha+i \beta|^{2}}-\frac{1}{|1+\alpha+i \beta|} \\
& =\frac{1}{|1+\alpha+i \beta|}\left(\frac{|-1+\alpha+i \beta|}{|1+\alpha+i \beta|}-1\right)
\end{aligned}
$$

and $|1+\alpha+i \beta| \geq|-1+\alpha+i \beta|$.

Since $\kappa_{2}>0, \psi$ is a quadratic function with positive leading coefficient, and $\psi(1)=\kappa_{2}+\kappa_{1}+\kappa_{0} \leq \kappa_{0}=\psi(0)$, it follows that

$$
\psi(x) \leq \max \{\psi(0) ; \psi(1)\}=\psi(0)=\kappa_{0} \quad(x \in[0,1])
$$


Thus from (4.2), (4.3) and (4.5) we obtain

$$
\left|a_{3}\right|-\left|a_{2}\right| \leq \kappa_{0}=\frac{2}{|2+\alpha+i \beta|}
$$

We next prove the lower bound in (4.1).

Write

$$
\left|a_{3}\right|-\left|a_{2}\right|=\frac{2}{|2+\alpha+i \beta|} \Psi,
$$

where

$$
\Psi=\left|R_{1} e^{i \theta} \zeta_{1}^{2}+\left(1-\zeta_{1}^{2}\right) \zeta_{2}\right|-R_{2} \zeta_{1}
$$

with

$$
R_{1}=\left|\frac{3+\alpha+i \beta}{(1+\alpha+i \beta)^{2}}\right|, \quad \theta=\arg \left(\frac{3+\alpha+i \beta}{(1+\alpha+i \beta)^{2}}\right)
$$

and

$$
R_{2}=\left|\frac{2+\alpha+i \beta}{1+\alpha+i \beta}\right|
$$

so that we need to show that

$$
\Psi \geq \frac{-R_{2}}{\sqrt{R_{1}+1}}
$$

Since both $\mathcal{B}_{1}(\alpha, i \beta)$ and $\mathcal{P}$ are rotationally invariant, we may assume that $\zeta_{1} \in$ $[0,1]$.

Now write $\zeta_{2}=s e^{i \varphi}$ with $s \in[0,1]$ and $\varphi \in \mathbb{R}$, so that

$$
\Psi=\left|R_{1} e^{i(\theta-\varphi)} \zeta_{1}^{2}+\left(1-\zeta_{1}^{2}\right) s\right|-R_{2} \zeta_{1} .
$$

Then

$$
\begin{aligned}
\Psi & =\sqrt{R_{1}^{2} \zeta_{1}^{4}+2 R_{1} \zeta_{1}^{2}\left(1-\zeta_{1}^{2}\right) s \cos (\theta-\varphi)+\left(1-\zeta_{1}^{2}\right)^{2} s^{2}}-R_{2} \zeta_{1} \\
& \geq\left|R_{1} \zeta_{1}^{2}-\left(1-\zeta_{1}^{2}\right) s\right|-R_{2} \zeta_{1},
\end{aligned}
$$

with equality when $\cos (\theta-\varphi)=-1$. 
Suppose that $R_{1} \zeta_{1}^{2}-\left(1-\zeta_{1}^{2}\right) s \leq 0$, then $\zeta_{1} \leq \sqrt{s /\left(R_{1}+s\right)}=: \eta_{1}$, and so by (4.6) it follows that

$$
\begin{aligned}
\Psi & \geq-\left(R_{1}+s\right) \zeta_{1}^{2}-R_{2} \zeta_{1}+s \\
& \geq-\left(R_{1}+s\right) \eta_{1}^{2}-R_{2} \eta_{1}+s \\
& =-R_{2} \sqrt{\frac{s}{R_{1}+s}} \\
& \geq \frac{-R_{2}}{\sqrt{R_{1}+1}}
\end{aligned}
$$

since $s \leq 1$.

If $R_{1} \bar{\zeta}_{1}^{2}-\left(1-\zeta_{1}^{2}\right) s \geq 0$, then $\zeta_{1} \geq \eta_{1}$, and define $\phi$ by

$$
\phi(x)=\left(R_{1}+s\right) x^{2}-R_{2} x-s,
$$

and let

$$
\eta_{2}=\frac{R_{2}}{2\left(R_{1}+s\right)}
$$

be the unique critical point of $\phi$. Then by (4.6) we have

$$
\Psi \geq \phi\left(\zeta_{1}\right)
$$

The condition $\eta_{2} \geq \eta_{1}$ is equivalent to the inequality $4 s^{2}+4 R_{1} s-R_{2}^{2} \geq 0$, which holds for $0 \leq s \leq \lambda$, where

$$
\lambda=\lambda_{\alpha, \beta}:=\frac{1}{2}\left(-R_{1}+\sqrt{R_{1}^{2}+R_{2}^{2}}\right) .
$$

It is easily seen that $\lambda<1$ since

$$
R_{2}^{2}=\frac{(2+\alpha)^{2}+\beta^{2}}{(1+\alpha)^{2}+\beta^{2}} \leq\left(\frac{2+\alpha}{1+\alpha}\right)^{2} \leq 4<4+R_{1},
$$

for $\alpha \geq 0$, and $\beta \in \mathbb{R}$.

We also note that $R_{2}-2 R_{1}<2$, since

$$
R_{2}-2 R_{1}<R_{2} \leq \frac{2+\alpha}{1+\alpha} \leq 2
$$

We consider next the case $R_{2} \leq 2 R_{1}$, where $\eta_{1} \leq 1$ for all $s \in[0,1]$, and distinguish two sub-cases, $\eta_{2} \leq \eta_{1}$, and $\eta_{2} \geq \eta_{1}$.

When $s \in[\lambda, 1]$, we have $\eta_{2} \leq \eta_{1}$, and so from (4.7) we obtain

$$
\Psi \geq \phi\left(\eta_{1}\right)=-R_{2} \sqrt{\frac{s}{R_{1}+s}} \geq \frac{-R_{2}}{\sqrt{R_{1}+1}}
$$


since $s \in[0,1]$. When $s \in[0, \lambda]$, we have $\eta_{2} \geq \eta_{1}$. This, and (4.7), implies that

$$
\Psi \geq \phi\left(\eta_{2}\right)=-\left(s+\frac{R_{2}^{2}}{4\left(R_{1}+s\right)}\right)=-\frac{1}{4} h(s),
$$

where $h$ is defined by

$$
h(x)=4 x+\frac{R_{2}^{2}}{R_{1}+x} .
$$

Differentiating $h$ gives

$$
\left(R_{1}+x\right)^{2} h^{\prime}(x)=4 x^{2}+8 R_{1} x+4 R_{1}^{2}-R_{2}^{2} .
$$

Since $4 R_{1}^{2}-R_{2}^{2}=\left(2 R_{1}+R_{2}\right)\left(2 R_{1}-R_{2}\right) \geq 0, h$ is increasing on the interval $[0, \lambda]$, and so from (4.9) we have

$$
\Psi \geq-\frac{1}{4} h(\lambda)=-\left(\lambda+\frac{R_{2}^{2}}{4\left(R_{1}+\lambda\right)}\right) .
$$

Next note that

$$
\frac{R_{2}}{\sqrt{R_{1}+1}} \geq \lambda+\frac{R_{2}^{2}}{4\left(R_{1}+\lambda\right)},
$$

since

$$
\lambda+\frac{R_{2}^{2}}{4\left(R_{1}+\lambda\right)} \leq \frac{R_{2} \sqrt{\lambda}}{\sqrt{R_{1}+\lambda}},
$$

provided $\sqrt{\lambda\left(R_{1}+1\right)} \leq \sqrt{R_{1}+\lambda}$ which is valid for all $\alpha \geq 0$ and $\beta \in \mathbb{R}$ since $\lambda<1$.

Thus it follows from (4.8), (4.11) and (4.12) that

$$
\Psi \geq \frac{-R_{2}}{\sqrt{R_{1}+1}}
$$

is true provided $R_{2} \leq 2 R_{1}$.

Next assume that $R_{2} \geq 2 R_{1}$. In this case there exists $s \in[0,1]$, such that $\eta_{2} \geq 1$.

Setting $\hat{\lambda}=\left(R_{2}-2 R_{1}\right) / 2$ it follows that $0<\hat{\lambda}<\lambda<1$.

When $s \in[\lambda, 1]$, we have $\eta_{2} \leq \eta_{1}$, and a similar method to that used in the case $R_{2} \leq 2 R_{1}$ gives

$$
\Psi \geq \frac{-R_{2}}{\sqrt{R_{1}+1}}
$$


When $s \in[\hat{\lambda}, \lambda]$, we have $\eta_{2} \geq \eta_{1}$, and so the function $h$, defined by (4.10), is increasing on $[\hat{\lambda}, \lambda]$ since

$$
\begin{aligned}
\left(R_{1}+x\right)^{2} h^{\prime}(x) & =4 x^{2}+8 R_{1} x+4 R_{1}^{2}-R_{2}^{2} \\
& \geq 4 \hat{\lambda}^{2}+8 R_{1} \hat{\lambda}+4 R_{1}^{2}-R_{2}^{2}=0 \quad(x \in[\hat{\lambda}, \lambda]) .
\end{aligned}
$$

Thus from (4.11) and (4.12), we have

$$
\Psi \geq-\frac{1}{4} h(\lambda) \geq \frac{-R_{2}}{\sqrt{R_{1}+1}}
$$

When $s \in[0, \hat{\lambda}]$, we have $\eta_{2} \geq 1$, which implies

$$
\Psi \geq \phi(1)=R_{1}-R_{2}
$$

Finally from (4.13), in order to establish the left hand inequality in (4.1), it is enough to show that

$$
\frac{R_{2}}{\sqrt{R_{1}+1}} \geq R_{2}-R_{1}
$$

Since

$$
R_{1}-R_{2}+\frac{R_{2}}{\sqrt{R_{1}+1}}=R_{1} R_{2}\left(\frac{1}{R_{2}}-\frac{1}{R_{1}+1+\sqrt{R_{1}+1}}\right)
$$

and since $R_{1}>0$ and $R_{2}>0,(4.14)$ is satisfied, if for $\alpha \geq 0$ and $\beta \in \mathbb{R}$

$$
\sqrt{R_{1}+1}>R_{2}-R_{1}-1
$$

Since

$$
R_{2}-R_{1}-1=\frac{1}{|1+\alpha+i \beta|}\left(|2+\alpha+i \beta|-|1+\alpha+i \beta|-\frac{|3+\alpha+i \beta|}{|1+\alpha+i \beta|}\right)
$$

and

$$
|2+\alpha+i \beta| \leq|1+\alpha+i \beta|+1<|1+\alpha+i \beta|+\frac{|3+\alpha+i \beta|}{|1+\alpha+i \beta|},
$$

it follows that $R_{2}-R_{1}-1<0<\sqrt{R_{1}+1}$, which establishes (4.15), and hence (4.14).

Thus the proof of the inequalities for $\left|a_{3}\right|-\left|a_{2}\right|$ is complete.

In order to show that the inequalities are sharp, first let the function $f_{1}$ be defined by (1.3) with $g(z)=z$ and $p(z)=\left(1+z^{2}\right) /\left(1-z^{2}\right)$. Then $f_{1} \in \mathcal{B}_{1}(\alpha, i \beta)$ with 


$$
f_{1}(z)=z+\frac{2}{2+\alpha+i \beta} z^{3}+\cdots
$$

Thus the upper bound in (4.1) is sharp.

$$
\text { Next put } \zeta_{1}=1 / \sqrt{R_{1}+1} \text {, and } \zeta_{2}=s e^{i \varphi} \text { with } s=1 \text { and } \varphi=\theta-\pi \text {. Then }
$$

$$
\Psi=\left|R_{1} e^{i(\theta-\varphi)} \zeta_{1}^{2}+\left(1-\zeta_{1}^{2}\right) s\right|-R_{2} \zeta_{1}=-\frac{R_{2}}{\sqrt{R_{1}+1}} .
$$

Since $\zeta_{1} \in \mathbb{D}$ and $\zeta_{2} \in \mathbb{T}$, it follows from Lemma 2.2 that the function $\hat{p}$ defined by

$$
\begin{aligned}
\hat{p}(z) & =\frac{1+\left(\zeta_{1} \zeta_{2}+\zeta_{1}\right) z+\zeta_{2} z^{2}}{1+\left(\zeta_{1} \zeta_{2}-\zeta_{1}\right) z-\zeta_{2} z^{2}} \\
& =\frac{\sqrt{R_{1}+1}+\left(e^{i \varphi}+1\right) z+\sqrt{R_{1}+1} e^{i \varphi} z^{2}}{\sqrt{R_{1}+1}+\left(e^{i \varphi}-1\right) z-\sqrt{R_{1}+1} e^{i \varphi} z^{2}}
\end{aligned}
$$

belongs to $\mathcal{P}$. Now let the function $f_{2}$ be defined by (1.3) with $g(z)=z$ and $p=\hat{p}$. Then $f_{2} \in \mathcal{B}_{1}(\alpha, i \beta)$. From (4.5) and (4.16), we obtain

$$
\left|a_{3}\right|-\left|a_{2}\right|=\frac{2}{|2+\alpha+i \beta|} \Psi=-\frac{2}{\sqrt{|1+\alpha+i \beta|^{2}+|3+\alpha+i \beta|}},
$$

which shows that the left hand equality in (4.1) is sharp.

This completes the proof of Theorem 4.1.

Acknowledgements The first author was supported by the Basic Science Research Program through the National Research Foundation of Korea (NRF) funded by the Ministry of Education, Science and Technology (No. 2019R1I1A3A0105086). The second author was supported by the National Research Foundation of Korea(NRF) Grant Funded by the Korea Government (MSIP; Ministry of Science, ICT \& Future Planning) (No. NRF-2017R1C1B5076778).

Open Access This article is distributed under the terms of the Creative Commons Attribution 4.0 International License (http://creativecommons.org/licenses/by/4.0/), which permits unrestricted use, distribution, and reproduction in any medium, provided you give appropriate credit to the original author(s) and the source, provide a link to the Creative Commons license, and indicate if changes were made.

\section{References}

1. Bazilevič, I.E.: On a case of integrability in quadratures of the Loewner-Kufarev equation. Mat. Sb. N. S. 37(79), 471-476 (1955)

2. De Branges, L.: A proof of the Bieberbach conjecture. Acta Math. 154(1-2), 137-152 (1985)

3. Cho, N.E., Kowalczyk, B., Lecko, A.: The sharp bounds of some coefficient functionals over the class of functions convex in the direction of the imaginary axis. Bull. Aust. Math. Soc. (to appear)

4. Duren, P.L.: Univalent functions. Springer, Amsterdam (1983)

5. Grinspan, A.Z.: The sharpening of the difference of the moduli of adjacent coefficients of schlicht functions. In: Some problems in modern function theory. In: Proceedings of conference modern problems of geometric theory of functions, Inst. Math., Acad. Sci. USSR, Novosibirsk, (Russian), Akad. Nauk SSSR Sibirsk. Otdel. Inst. Mat., Novosibirsk, pp. 41-45 (1976)

6. Hayman, W.K.: On successive coefficients of univalent functions. J. Lond. Math. Soc. 38, 228-243 (1963) 
7. Koepf, W.: On the Fekete-Szego problem for close to convex functions. Proc. Am. Math. Soc. 101, 89-95 (1987)

8. Leung, Y.: Successive coefficients of starlike functions. Bull. Lond. Math. Soc. 10, 193-196 (1978)

9. Ma, W.C., Minda, D.: A unified treatment of some special classes of univalent functions. In: Proceedings of the Conference on Complex Analysis, Tianjin, 1992. Conf. Proc. Lecture Notes Anal., vol. I, pp. 157-169. International Press, Cambridge, (1992)

10. Sheil-Small, T.: On Bazilevič functions. Quart. J. Math. Oxf. Ser. 2(23), 135-142 (1972)

11. Singh, R.: On Bazilevič functions. Proc. Am. Math. Soc. 38(2), 261-271 (1973)

Publisher's Note Springer Nature remains neutral with regard to jurisdictional claims in published maps and institutional affiliations. 